\title{
Utility of Diffusion-weighted Imaging for Characterization and Diagnosis of Liver Lesions
}

\section{Zeynab Yazdi Sotoodeh ${ }^{\text {*, }}$ Vida Sargazi ${ }^{1}$ M. Farsizaban $^{2}$}

'Department of Radiology, Faculty of Paramedicine, Zahedan University of Medical Science, Zahedan, Iran

${ }^{2}$ Department of Medical Physics, Zahedan University of Medical Science, Zahedan, Iran

Study Area:Zahedan, Iran

Coordinates: $29^{\circ} 29^{\prime} 47^{\prime \prime} \mathrm{N} ; 60^{\circ} 51^{\prime} 46^{\prime \prime} \mathrm{E}$

Key words: Prospective clinical trial,DWI

\begin{abstract}
Diffusion weighted imaging provides qualitative and quantitative information about the diffusion properties. It adds a new dimension to the magnetic resonance imaging (MRI) examinations by adding functional information to the largely anatomical information gathered by the conventional sequences. DWI uses is increasing over the past few years in extracranial organs including the abdomen and pelvis. Although further studies are needed to determine its performance in evaluating bile duct, gallbladder, pancreas and Liver diseases, DWI has potential in the assessment of the functional information on the Liver concerning the status of tissue cellularity. It is just because increased cellularity is associated with impeded diffusion, as indicated by a reduction in the apparent diffusion coefficient. DWI should be included as a basic sequence in the routine MRI study of the liver as it helps in diagnosis and so reaching a final diagnosis or at least trying to narrow the list of differential diagnosis .
\end{abstract}

\section{Introduction:}

MRI plays an important role in the evaluation of patients suffering from liver disease due to its high contrast resolution, lack of ionizing radiation, and the possibility of performing functional imaging sequences With advances in hardware and coil systems, diffusion weighted imaging (DWI) can now be applied to liver imaging with improved image quality (Qayyum, 2009; Taouli \& Koh, 2010).

Diffusion-weighted magnetic resonance imaging (DWI) provides information on the random (Brownian) motion of water molecules in the body. The degree of restriction to water diffusion in biological tissues is directly proportional to tissue cellularity and the integrity of cell membranes. With the continuous development and improvement of hardware and software of magnetic resonance imaging (MRI), the use of parallel acquisition techniques to reduce scan time and improve spatial resolution, and the wide application of the shortened echo chain technique in spin-echo echo-planar imaging (SEEPI), diffusion-weighted imaging (DWI) has been increasingly used in a wide range of clinical applications (Bammer \& Schoenberg, 2004; Koike et al. 2009). The degree of water motion is found to be proportional to the degree of signal attenuation in DWI. Thus, more cellular solid tumors show relatively higher signal intensities and exhibit lower apparent diffusion coefficient (ADC, expressed in $\mathrm{mm} 2 / \mathrm{s}$ ) values on DWI using two or more b values than do less cellular tissues. Recently, this utility of DWI has been expanded to examination of abdominal organs; the ADC values of malignant masses are signif icantly lower than those of benign masses in the liver, pancreas, kidney, and prostate, although there is a small degree of overlap (Taouli et al., 2003; Parikh et al., 2008; Fattahi etal. 2009; Saremi et al., 2009 \& Zhang et al., 2008).

\section{Basic concept:}

Pathological conditions, such as tumors, cytotoxic edema, abscesses, and fibrosis, in which the physical nature of the intracellular and extracellular spaces changes, result in increased restriction of the diffusion of water molecules. In tissues of low cellularity or where the cellular membranes have been disrupted, the diffusion of water molecules is relatively free or less restricted (Fig.-1) (Koh \& Collins, 2007; Qayyum, 2009; Thoeny et al., 2013; Saremi et al., 2009; Patterson et al., 2008).

Within biologic tissues, the movement of water is not completely random, but rather, is impeded by the interaction with tissue compartments, cell membranes, and intracellular organelles. For purposes of simplification, water movement in tissues may be categorized as intravascular, intracellular, or extracellular (Fig.-1). (Qayyum, 2009; Patterson ,2008). 


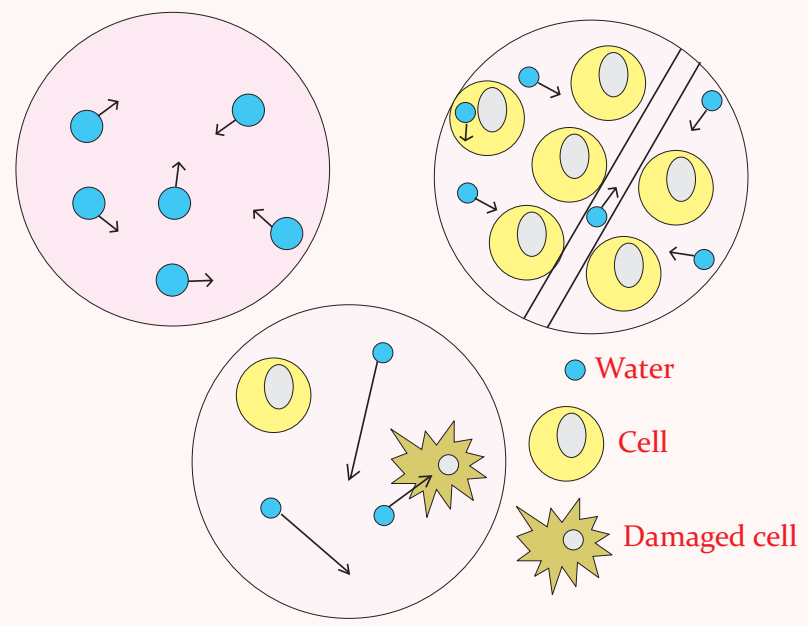

Figure-1: Schematic illustrating water molecule movement. In (A) water molecules in a container alone move randomly (Brownian motion). In (B) highly cellular tissue impedes the movement of water molecules. Their movement can be categorized as intravascular, intracellular, or extracellular. In (C) tissue of low cellularity or with defective cells permits greater water molecule movement. Tissues with low cellularity or that consist of cells with disrupted membranes permit greater movement of water molecules.

The sensitivity of a DWI sequence to water diffusion can be altered by changing the parameter known as the $b$ value, which represents the diffusion factor (measured in s/mm2) and the strength of the diffusion gradients. DWI is obtained with at least two different $b$ values. The images obtained at different $b$ values allow the quantification of the ADC of tissues, which is usually displayed as a parametric (ADC) map. The mean or median ADC value can be measured by drawing regions of interest (ROIs) on the ADC map. Tissues with restricted diffusion and high cellular density show low $A D C$ values, whereas tissues with less cellular density show higher ADC values (Koh \& Collins 2007; Qayyum, 2009).

The signal intensity of DWI is dependent on the diffusion of water molecules and $\mathrm{T}_{2}$ relaxation time. Thus, an area with a very long $T 2$ relaxation time (e.g., bile in the biliary tract) maintains high signals on high-b-value DWI, and can be mistaken for restricted diffusion. This phenomenon is known as the "T2 shine-through" effect (Plate-2). The T2 shine-through effect inevitably confounds DWI images, and the ADC map eliminates this effect. Moreover, because this technique is usually performed using a single-shot EPI technique, the signal-to-noise ratio and lesion conspicuity are reduced. In contrast, respirationtriggered DWI provides higher signal-to-noise and contrast-to-noise ratios, although its acquisition time is longer than that of breath-hold DWI (Koh et al., 2007 \& Kandpal et al., 2009).

Unlike brain imaging where $b$ values are well established, b values in other parts of the body vary widely between investigators. Thus, b values for body imaging require optimization.

\section{Choice of bvalues \& sequence optimization:}

Because of the relatively short $\mathrm{T} 2$ relaxation time of the normal liver parenchyma, the $\mathrm{b}$ values used for clinical imaging are typically no higher than $1000 \mathrm{~s} / \mathrm{mm}^{2}$. Applying a small diffusion weighting of $\mathrm{b}$ less than $100-150 \mathrm{~s} / \mathrm{mm}^{2}$ nulls the intrahepatic vascular signal, creating the so-called black-blood images, which improve the detection of focal liver lesions (Plate-2) while higher b values $\left(\mathrm{P}_{500 \mathrm{~s}} / \mathrm{mm}^{2}\right)$ give diffusion information that helps in focal liver lesion characterization. Hence, when performing DW MR imaging in the liver, it is advantageous to perform imaging by using both lower and higher b values (e.g., b $\mathrm{P}_{50}$, b P1oo, \& b P $500 \mathrm{~s} / \mathrm{mm}^{2}$ ) (Parikh et al., 2008; Hussain et al., 2005; Van den Bos \& Wielopolski, 2008; Taouli et al., 2003). Quantitative analysis of diffusion-weighted imaging findings can be performed only if at least two b values are used for imaging (Taouli et al., 2007; Taouli et al., 2008; Chandarana \& Taouli, 2010). In clinical practice, higher-bvalue DWI (usually $800-1000 \mathrm{~s} / \mathrm{mm} 2$ ) results in a reduction of the signal from moving protons in the bile ducts, cyst, vessels, and fluid in the bowel. This leads to a reduction in the $\mathrm{T} 2$ shine-through effect, resulting in increased contrast between lesions and visceral organs such as the liver or gallbladder. However, the tradeoffs of higher-b-value DWI are a lower signal-to-noise ratio, the possibility of ADC error, and increased image distortion due to the longer echo time required (Koh et al., 2007; Kandpal et al., 2009; Whittaker et al., 2009; Saremi et al., 2009). ADC measurements are then recorded for a given region by drawing regions of interest on the ADC Map (Qayyum, 2009; Taouli, 2010; Boll \& Merkle, 2009; Berman et al., 2004; Furukawa et al., 2001; \& Takeuchi et al., 2003).

\section{Evaluation Liver lesion:}

DWI is helpful in focal liver lesion detection and characterization and can be used as an alternative to Gadolinium-enhanced MRI in patients with Liver dysfunction (Chan et al., 2001). Clinical applications of DW MRI include treatment response monitoring and prognostication in patients receiving systemic and focal ablative therapies for hepatic and pancreatic malignancies (De Robertis et al., 2015; Cuneo et al., 2014; Kurosawa et al., 2015; Mannelli et al., 2013). Hardie et al. (2010) compared the utility of DWI in detection of liver metastases. They reported that DWI has $66.3 \%$ sensitivity compared to $73.5 \%$ for CE-MRI and hence it can serve as a useful alternative for this purpose.

DW MR imaging has been investigated in diffuse hepatic parenchymal diseases such as non-alcoholic fatty liver disease and hepatic fibrosis. However, its clinical applicability is questionable as it suffers from multiple confounders making quantifying liver fibrosis through ADC diff icult (Hong et al., 2015).

Hepatic abscesses are def ined as intrahepatic single or multiple collections of pus within the liver. Those result 
from infectious cholangitis and are usually seen in patients with suppurative cholangitis. Pyogenic abscesses have variable signal intensities on T1- and T2-weighted images. Abscesses can be a life-threatening emergency if not recognized and treated timely (Méndez et al., 1994).

DWI is helpful in the early detection of abscesses and their differentiation from cystic or necrotic tumors. On DWI using high b values, abscesses appear hyperintense with low ADC values (Plate-1) (Chan et al., 2001).

Diffuse periductal thickening along the bile duct in the periductal-infiltrating type, causing mild-to-marked bile duct dilatation, is seen in intrahepatic cholangiocarcinoma (Chung et al., 2001). Mass-forming intrahepatic cholangiocarcinoma is usually large, achieving a diameter of up to 15 $\mathrm{cm}$ because early symptoms are rarely present. Thus, it is readily depicted on DWI and remains hyperintense on DWI using high b values with low ADC values; similar to values of other malignant focal hepatic lesions (Plate-2).

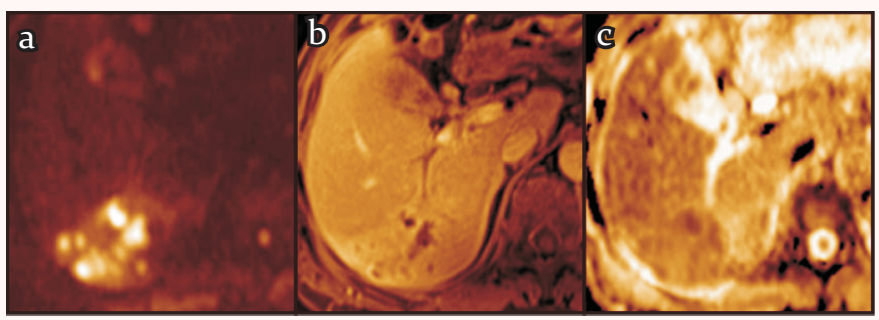

Plate-1: Liver abscesses complicating acute cholangitis in a 79-yearold man. a: DWI at $\mathrm{b}=1000 \mathrm{~s} / \mathrm{mm}^{2}$ shows multiple liver abscesses with high signal intensity (arrowheads); b: Multiple abscesses with peripheral rim enhancement (arrowheads) are less conspicuous on contrast-enhanced fat-saturated $\mathrm{T}_{1}$ weighted images (B) than on DWI (A); c: On an ADC map, multiple abscesses appear as low signal intensity (arrowheads) due to restriction of diffusion. DWI: Diffusion-weighted magnetic resonance imaging; ADC: Apparent diffusion coefficient. DWI using high b values can be useful for the detection of cholangiocarcinoma associated to hepatolithiasis

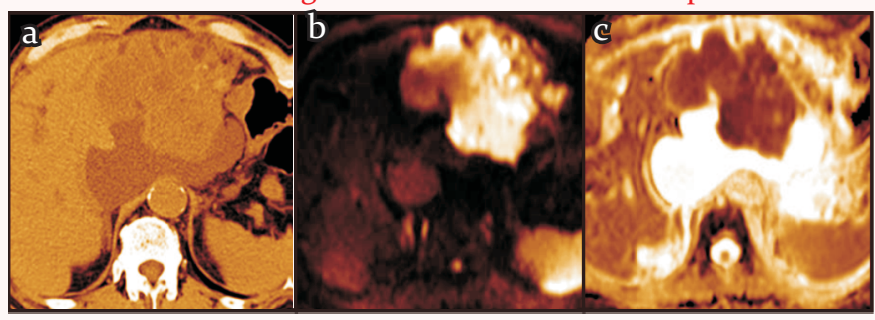

Plate-2: Intrahepatic cholangiocarcinoma (mass-forming type) in a 71-year-old man. a: Nonenhanced CT image shows intrahepatic cholangiocarcinoma in the left lobe of the liver (asterisk), stones in the dilated left bile ducts (arrow), and a fluid collection in the lesser sac (arrowhead); b: DWI at b $=1000 \mathrm{~s} / \mathrm{mm}^{2}$ shows intrahepatic cholangiocarcinoma with high signal intensity (asterisk). Note a fluid collection (arrowhead) in the lesser sac, which appears as significant attenuation of the signal intensity reduction at a high $b$ value; c: On the ADC map, cholangiocarcinoma appears as low signal intensity (asterisk) due to restriction of diffusion. DWI: Diffusion-weighted magnetic resonance imaging; CT: Computed tomography;
ADC: Apparent diffusion coefficient. Central hypointensity of the mass lesion may be seen on T2-weighted images and DWI using high $b$ values, which may reflect fibrotic tissue in the central portion of the tumor. DWI may be helpful for the detection of malignant bile duct lesions causing a variable degree of intrahepatic bile duct dilatation, as well as for differentiating between intrahepatic malignant and benign bile duct lesions (Plate-3).

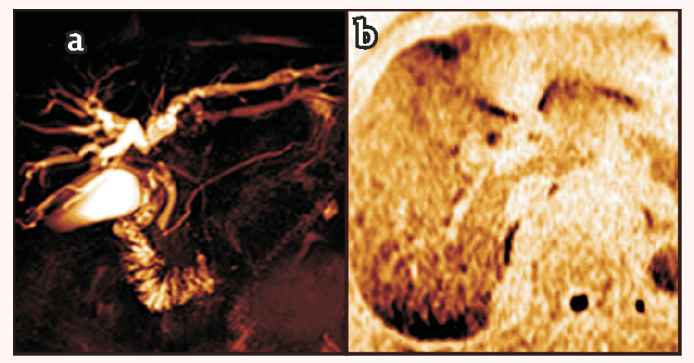

Figure 4: Cholangiocarcinoma (periductal-infiltrating type) in a 70-yearold man. a: Coronal image from thick-slab, single-shot MRCP shows marked dilatation of the intrahepatic ducts and abrupt narrowing at the confluence of the hepatic duct (arrow); b: DWI at b $=800 \mathrm{~s} / \mathrm{mm}^{2}$ with inverted black-andwhite image contrast clearly depicts the mass (arrow) at the confluence of the hepatic duct. DWI: Diffusion-weighted magnetic resonance imaging; MRCP: Magnetic resonance cholangiopancreatography

\section{Discussion:}

TMRI of the liver is an important tool for the detection and characterization of focal liver lesions. With recent advances in technology, functional MRI methods such as DW is increasingly used in the abdomen with promising results, particularly in the evaluation of focal liver diseases (Sun et al., 2005; Chandarana \& Taouli, 2010; Parikh et al., 2008). Although MRI is more superior and less hazardous than CT in the diagnosis of hepatic lesions, it needs a cooperative patient during a relatively long time of examination while compared with CT and also it is more expensive than CT Images with higher $b$ values provide diffusion information that aids in lesion characterization.18,41 Malignant lesions have lower mean ADC values than benign lesions, with varying degrees of overlap (Sun et al., 2005; Parikh et al., 2008; Gourtsoyianni et al., 2008).

There are few technical limitations of diffusionweighted imaging in the liver are also exist in it. As DWI is susceptible to a variety of artifacts that arise from motion, physiological motion artifacts such as respiratory motion, cardiac pulsation, movement of the diaphragm, and motility of the bowel lead to ghosting images and blurring. The pulsatile motion of the heart and aorta obscure or diminish visualization of and increase ADC in the left lobe of the liver. These artifacts can be overcome using respiratory or electrocardiographic triggering. The EPI technique produces a low spatial resolution and signal-tonoise ratio. The rapid on and off switching of the highintensity gradient field easily produce eddy currents, 
leading to geometrical distortion and image shearing artifacts that may become more pronounced with increased b values. DWI is also highly sensitive to magnetic field inhomogeneity (Koh et al., 2007; Le Bihan et al., 2006).

detection of small malignant bile duct lesions is limited on DWI because tumor detection with DWI is affected by variable causes, such as a low spatial resolution of higher-bvalue DWI, tumor cellularity, bowel peristalsis,

and artifacts (Wang et al., 1999).

Furthermore, there remain substantial technical hurdles, even though the rapid evolution of MRI systems is making ever more powerful approaches possible. Such improvements are particularly welcome given the limited signal-to-noise ratio of diffusion overall. Nevertheless, these initial data are promising (Guye et al., 2003).

\section{Conclusion:}

DWI reflects the micro-movements of water molecules. It produces images by comparing the inter-tissue diffusion coefficient. The composition of tissues, and the functional status of water exchange between tissue components affected by pathological or physiological status, given the fact that DWI is inherently T2-weighted. An extra-long T2 relaxation time can also show as continuously higher signal intensity in DWI, which may then be misdiagnosed as restricted diffusion. This is known as the T2 shine-through effect (Roberts et al., 2003; Castillo, 2003). To avoid this effect, ADC values can be calculated using two images obtained under different b values. The ADC map can then be used to overcome the T2 shine-through effect by observing the relative reduction in signal in images obtained under different $b$ values. DWI is now widely applied to the detection and conf irmation of cancer in liver, pancreas, kidney, colon, and prostate (Parikh et al., 20o8; Matsuki et al., 2007; Zhang et al., 2007; Ichikawa et al., 2006; Issa, 2002).

DWI is an important complementary sequence with the routine MRI of the liver that helps in lesion quantification and characterization in a hope to reach a final confident diagnosis and in order to reduce the rate of biopsy. Although the MRI is a safe technique without exposure to radiation it has some disadvantages as the examination takes a longer time than in CT and it is also more expensive than CT. Therefore, MRI and DWI should be used in selected lesions where their use would add benef it to the patient.

\section{References:}

Bammer, R. \& Schoenberg, S.O. (2004): Current concepts and advances in clinical parallel magnetic resonance imaging. Top Magn. Reson. Imaging, 15(3):129-158.

Berman, J.I., Berger, M.S., Mukherjee, P. \& Henry, R.G. (2004): Diffusion tensor imaging-guided tracking of fibers of the pyramidal tract combined with intraoperative cortical stimulation mapping in patients with gliomas. J. Neurosurg., 101(1):66-72.

Bruegel, M., Gaa, J., Waldt, S., Woertier, K., Holzapfel , K., Kiefer, B.
\& Rummeny, E.J. (2008): Diagnosis of hepatic metastasis: comparison of respiration-triggered diffusion-weighted echo-planar MRI and five T2-weighted turbo spin-echo sequences. Am. J. Roentgenol., 191(5):1421-1429.

Bruegel, M., Holzapfel, K., Gaa, J., Woertler, K., Waldt, S., Kiefer, B., Stemmer, A., Ganter, C. \& Rummeny, E.J. (2008): Characterization of focal liver lesions by ADC measurements using a respiratory triggered diffusionweighted single-shot echoplanar MR imaging technique. Eur. Radiol., 18(3):477-485.

Castillo, M. (2003): Diffusion-weighted imaging of the spine: is it reliable? Am. J. Neuroradiol., 24 (6):1251-1253.

Chan, J.H., Tsui, E.Y., Luk, S.H., Fung, A.S., Yuen, M.K., Szeto, M.L., Cheung, Y.K. \& Wong, K.P. (2001): Diffusion-weighted MR imaging of the liver: distinguishing hepatic abscess from cystic or necrotic tumor. Abdom. Imaging, 26 (2):161-165.

Chandarana, H. \& Taouli, B. (2010): Diffusion and perfusion imaging of the liver.Eur. J. Radiol., 76(3):348-358.

Chung, Y.E., Kim, M.J., Park, Y.N., Choi, J.Y., Pyo, J.Y., Kim, Y.C., Cho, H.J., Kim, K.A. \& Choi, S.Y. (2009): Varying appearances of cholangiocarcinoma: radiologic-pathologic correlation. Radiographics, 29(3):683-700.

Cuneo, K.C., Chenevert, T.L., Ben-Josef, E., Feng, M.U., Greenson, J.K., Hussain, H.K., Simeone, D.M., Schipper, M.J., Anderson, M.A., Zalupski, M.M., Al-Hawary, M., Galban, C.J., Rehemtulla, A., Feng, F.Y., Lawrence, T.S. \& Ross, B.D. (2014): A pilot study of diffusion-weighted MRI in patients undergoing neoadjuvant chemoradiation for pancreatic cancer. Transl. Oncol., 7(5):644-649.

De Robertis, R., Tinazzi Martini, P., Demozzi, E., Puntel, G., Ortolani, S., Cingarlini, S., Ruzzenente, A., Guglielmi, A., Tortora, G., Bassi, C., Pederzoli, P. \& D'Onofrio, M. (2015): Prognostication and response assessment in liver and pancreatic tumors: The new imaging. World J. Gastroenterol., 21(22):6794-68o8.

Boll, D.T. \& Merkle, E.M. (2009): Diffuse liver disease: strategies for hepatic CT and MR imaging. Radiographics, 29(6):1591-1614.

Fattahi, R., Balci, N.C., Perman, W.H., Hsueh, E.C., Alkaade, S., Havlioglu, N. \& Burton, F.R. (2009): Pancreatic diffusionweighted imaging (DWI): comparison between massforming focal pancreatitis (FP), pancreatic cancer (PC), and normal pancreas. L. Magn. Reson. Imaging, 29(2):350-356.

Furukawa, M.K.K., Kamada, H. \& Miyasaka, K. (2001): MRI in seven cases of tacrolimus (FK-506) encephalopathy: utility of FLAIR and diffusion-weighted imaging. Neuroradiology, 43(8):615-621.

Gourtsoyianni, S., Papanikolaou, N., Yarmenities, S., Maris, T., Karantanas, A. \& Gourtsoyiannis, N. (2008): Respiratory gated diffusion weighted imaging of the liver: value of apparent diffusion coefficient measurements in the differentiation between most commonly en-countered benign and malignant focal liver lesions. Eur. Radiol., 18(3):486-492.

Guye, M., Parker, G.J.M., Symms, M., Boulby, P., WheelerKingshott, C.A.M., Salek-Haddadi, A., Barker, G.J. \& Duncan, J.S. (2003): Combined functional MRI and tractography to demonstrate the connectivity of the human primary motor cortex in vivo. Neuroimage, 19(4):1349-1360.

Hardie, A.D., Naik, M., Hecht, E.M., Chandarana, H., Mannelli, L., Babb, J.S. \& Taouli, B. (2010): Diagnosis of liver metastases: 


\section{REVIEW ARTICLE}

value of diffusionweighted MRI compared with gadoliniumenhanced MRI. Eur. Radiol., 20(6):1431-1441.

Hong, B.Z., Li, X.F. \& Lin, J.Q. (2015): Differential diagnosis of pancreatic cancer by single-shot echo-planar imaging diffusion-weighted imaging. World J. Gastroenterol., 21(20):6374-6380.

Hussain, S.M., De Becker, J., Hop, W.C., Dwarkasing, S. \& Wielopolski, P.A. (2005): Can a single-shot black-blood T2weighted spin-echo echo planar imaging sequence with sensitivity encoding replace the respiratory-triggered turbo spin-echo sequence for the liver? An optimization and a feasibility study. L. Magn. Reson. Imaging, 21(3):219-239.

Ichikawa, T., Erturk, S.M., Motosugi, U., Sou, H., Iino, H., Araki, T. \& Fujii, H. (2006): High-B-value diffusion-weighted MRI in colorectal cancer. Am. J. Roentgenol., 187(1):181-184.

Issa, B. (2002): In vivo measurement of the apparent diffusion coefficient in normal and malignant prostatic tissues using echoplanar imaging. J. Magn. Reson. Imaging, 16(2):196-200.

Kandpal, H., Sharma, R., Madhusudhan, K.S. \& Kapoor, K.S. (2009): Respiratory-triggered versus breath-hold diffusionweighted MRI of liver lesions: comparison of image quality and apparent diffusion coeff icient values. Am. J. Roentgenol., 192(4):915-922.

Koh, D.M. \& Collins, D.J. (2007): Diffusion-weighted MRI in the body: applications and challenges in oncology. Am. J. Roentgenol., 188(6):1622-1635.

Koh, D.M., Takahara, T., Imai, Y. \& Collins, D.J. (2007): Practical aspects of assessing tumors using clinical diffusion-weighted imaging in the body. Magn. Reson. Med. Sci., 6(4):211-224.

Koike, N., Cho, A., Nasu, K., Seto, K., Nagaya, S., Ohshima, Y., Nobuhiro, O. (2009): Role of diffusion-weighted magnetic resonance imaging in the differential diagnosis of focal hepatic lesions. World J. Gastroenterol., 15(46):5805-5812.

Kurosawa, J., Tawada, K., Mikata, R., Ishihara, T., Tsuyuguchi, T., Saito, M., Shimofusa, R., Yoshitomi, H., Ohtsuka, M., Miyazaki, M. \& Yokosuka, O. (2015): Prognostic relevance of apparent diffusion coefficient obtained by diffusionweighted MRI in pancreatic cancer. J. Magn. Reson. Imaging, 42(6):1532-1537.

Le Bihan, D., Poupon, C., Amadon, A. \& Lethimonnier, F. (2006): Artifacts and pitfalls in diffusion. L. Magn. Reson. Imaging, 24(3):478-488.

Mannelli, L., Bhargava, P., Osman, S.F., Raz, E., Moshiri, M., Laffi, G., Wilson, G.J., Maki, J.H. (2013): Diffusion-weighted imaging of the liver: a comprehensive review. Curr. Probl. Diagn. Radiol., 42(3):77-83.

Méndez, R.J., Schiebler, M.L., Outwater, E.K. \& Kressel, H.Y. (1994): Hepatic abscesses: MR imaging findings. Radiology, 190(2):431-436.

Matsuki, M., Inada, Y., Nakai, G., Tatsugami, F., Tanikake, M., Narabayashi, I., Masuda, D., Arisaka, Y., Takaori, K. \& Tanigaw, N. (2007): Diffusion-weighed MR imaging of pancreatic carcinoma. Abdom. Imaging, 32(4):481-483.

Parikh, T., Drew, S.J., Lee, V.S., Wong, S., Hecht, E.M., Babb, J.S. \& Taouli, B. (2008): Focal liver lesion detection and characterization with diffusion-weighted MR imaging: comparison withstandard breath-hold $\mathrm{T}_{2}$-weighted imaging. Radiology, 246C(3): 812-822.
Ambient Science, 2019: Vol. 06h(1); 01-05 DOI:10.21276/ambi.2019.06h.1.rv01

Patterson, D.M., Padhani, A.R. \& Collins, D.J. (2008): Technology insight: water diffusion MRI--a potential new biomarker of response to cancer therapy. Nat.Clin.Pract.Oncol., 5(4):220-233.

Qayyum, A. (2009): Diffusion-weighted imaging in the abdomen and pelvis: concepts and applications. Radiographics, 29(6):1797-1810.

Roberts, T.P. \& Schwartz, E.S. (2007): Principles and implementationof diffusion-weighted and diffusion tensor imaging. Pediatr. Radiol., 37(8):739-748.

Saremi, F., Knoll, A.N., Bendavid, O.J., Schultze-Haakh, H., Narula, N. \& Sarlati, F. (2009): Characterization of genitourinary lesions with diffusion-weighted imaging. Radiographics, 29 (5):1295-1317.

Sun, X-J., Quan, X-Y., Huang, F-H. \& Xu, Y-K. (2005):Quantitative evaluation of diffusion-weighted magnetic resonance imaging of focal hepatic lesions. World J. Gastroenterol. $11(41): 6535-3537$.

Takeuchi, M., Harada, M., Hisaoka, S., Nishitani, H., Mori, K. \& Sakama, M. (2003): Magnetic resonance imaging and proton MR spectroscopy of the brain in a patient with carbohydrate deficient glycoprotein syndrome type I. L. Magn. Reson. Imaging, 17(6):722-725.

Taouli, B., Chouli, M., Martin, A.J., Qayyum, A., Coakley, F.V. \& Vilgrain, V. (2008): Chronic hepatitis: role of diffusionweighted imaging and diffusion tensor imaging for the diagnosis of liver fibrosis and inflammation. J. Magn. Reson. Imaging, 28(1):89-95

Taouli, B. \& Koh, D-M. (2010): Diffusion-weighted MR Imaging of the liver. Radiology, 254(1):47-66.

Taouli, B., Tolia, A.J., Losada, M., Babb, J.S., Chan, E.S., Bannan, M.A. \& Tobias, H. (2007): Diffusion-weighted MRI for quantif ication of liver fibrosis: preliminary experience. Am. J. Roentgenol., 189(4):799-806.

Taouli, B., Vilgrain, V., Dumont, E., Daire, J.L., Fan, B., Menu, Y. (2003): Evaluation of liver diffusion isotropy and characterization of focal hepatic lesions with two single-shot echo-planar MRimaging sequences: prospective study in 66 patients. Radiology, 226(1): 71-78.

Thoeny, H.C. \& De Keyzer, F. (2007): Extracranial applications of diffusion-weighted magnetic resonance imaging. Eur. Radiol., $17(6): 1385-1393$

Van den Bos, I.C., Hussain, S.M., Krestin, G.P. \& Wielopolski, P.A. (2008): Liver imaging at $30 \mathrm{~T}$ :diffusion induced black-blood echo-planar imaging with large anatomic volumetric coverage as an alternative for specific absorption rateintensive echo-train spin-echo sequences: feasibility study. Radiology, 248(1):264-271.

Wang, H.J., Kim, J.H., Kim, J.H., Kim, W.H. \& Kim, M.W. (1999): Hepatocellular carcinoma with tumor thrombi in the bile duct. Hepatogastroenterol., 46(28): 2495-2499.

Whittaker, C.S., Coady, A., Culver, L., Rustin, G., Padwick, M. \& Padhani, A.R. (2009): Diffusion-weighted MR imaging of female pelvic tumors: a pictorial review. Radiographics, 29(3):759-774.

Zhang, J., Tehrani, Y.M., Wang, L., Ishill, N.M., Schwartz, L.H. \& Hricak, H. (2008): Renal masses: characterization with diffusionweighted MR imaging--a preliminary experience. Radiology. 247(2):458-464. 